\title{
Searching of Images Based on Content Using Blobs
}

\author{
Yaghoub Karimilivari ${ }^{1}$, Ladan Vasebi $^{1}$, Solmaz Vasebi ${ }^{2}$ \\ ${ }^{1}$ Sama Technical and Vocational Training College, Islamic Azad University, Sarab Branch, Sarab, Iran; ${ }^{2}$ Islamic Azad University, Sarab \\ Branch, Sarab, Iran. \\ Email: \{Y_ka76, Vasebi_la, Vasebi_so\}@yahoo.com
}

Received July $13^{\text {th }}, 2011$; revised December $8^{\text {th }}, 2011$; accepted January $12^{\text {th }}, 2012$

\begin{abstract}
Now a day's image searching is still a challenging problem in content based image retrieval (CBIR) system. Most system operates on all images without pre-sorting the images. The image search result contains many unrelated image. The aim of this research is to propose a new method for content based image indexing and research based on blobs feature extraction and existing edges in the image and classification of image to different type and to search image which are similar the given research.
\end{abstract}

Keywords: Indexing; Retrieval; Blob; Classification; Neural Network; Image; Edge

\section{Introduction}

In the recent year's production of large collection of digital data like image, video and etc. and advances in computer science and the growing information technology, there is an evident need for the development of indexing and retrieval systems. In recent decade many problems of content based system are considered and several solutions are offered. But ideal system must be proportional with the user point of view and permit to intuition search. Also indexing of content-base image is one of the important categories in information retrieval from multimedia databases. Image store in large databases, so image retrieval by content attracted more attention.

Furthermore, image database users generally want to find images based in subject that they observe, not only on the basis of name or low level features which images are common in that features. Most of the retrieval systems tend to perform fast quarry, but don't attention to image quality and to this that found images how are similar to observed image.

With the study of research's we found that there are many ambiguous points in image semantic indexing. Many questions that answer to each of them need a comprehensive research. As observed, in this research there is less attention to the user's point of view and to important features and further dealing with image's low level features or image's general features. The aim of this research is studing the effect of using existent blob in image and their feature in image indexing. However the effect of edge detection, histogram and neural network on indexing is studied.

An important property of this system is as follow:
- There is no need to exact specify objects boundary.

- Instead of a pixel, blobs are our comparison tool.

- Recognition of images which blobs are combined is possible due to use of relationship feature vector between edges.

- Using of color histogram cause to retrieval to be not turning.

- High speed of algorithm because of using classification.

- Acceptable results.

We continue this paper with previous works in image indexing and retrieval. In Section 3 we have a brief review on presented method. In Section 4 there is conclusion and the final section presents the totaling.

\section{Previous Work}

Most of the systems, do image retrieval based on image low level features and some of image indexing systems work with the general properties of image.

Method [1] detects similar regions in images and then compute feature vector with some region information such as level, angle, pattern and etc. This method is capable but ever segmentation is incomplete. Methods [2,3] act on colour segmentation that tend to find correctly objects boundary. Method [4] performs retrieval based on image segmentation regions. Segmentation of this method is not totally automatic. Method [5] is a system for image reieval incoherent regions of image. Each image with color and pattern description is automatically classified in regions. The blob world approach models blobs as grouped pixels in a mixture of Gaussian, using Expectation maximization. David Gavilan Ruiz proposes 
a system that work with blobs. This system is faster than prior but has the same problem and is sensitive to image rotation. Leung and Zheng present a method that use approximate segmentation in order to prevention of complete segmentation of estimable overhead. This method converted the image into a binary image, extracted shape factors, and employed them as a feature vector. Since a binary image cannot detect homogeneous regions perfectly and regions merge together, this method is ineffective particularly in large image data bases. Jain and Vailaya [6,7] introduced edge direction histogram. This method finds the image edges and with grouping them on the quantized edge direction, generates the EDH. This method is relatively effective and performs retrieval independent of translation, scaling, and small viewing position variations. Since this method uses the edges individually and ignores correlation between neighboring edges, its effectiveness is limited.

Sudirman and Qiu [8] introduce a method in which the color images, first appears as a combination of binary images which each one records the presence or absence of pre-defined factor like color, then visual binary algorithms are being used to analyze the geometric features of image which are independent of changeable graphic programs. In this query, cbst introduced as a portrayer of content Based image. In other word, this method is a simple mode of Blob world. The proposal scheme [9] is based on the analyzing of multi illumination image and colorful histogram. According to this approach, the indices of stroke wavelet image computed by employing a direct wavelet conversion like Gabor wavelet, and then one quantized stage is employed before calculating of auto correlogram and finally index vector is produced by using this mono directional corrilogram.

\section{Method Overview}

The method presented in this paper, following approaches which use the blobs for indexing. In this method, by employing edge orientation vector and color histogram, based on the segmentation of blobs, we both extract the blobs and ward off the existing problems in indexing methods. This problem exists just in figures in which the images ore merged or rotated. At first, by using feature vector auto correlogram (EOCA) $[10,11]$ we extract the correlations between edges existed in the image. And then get the existing blobs and store their feature vectors containing color, blob volume, position and etc in the database and finally store the color histogram, related to the image which is extracted from blobs, also in the database. After these steps, using MLP network we start by grouping blobs and then we use this grouping for clustering [12]. After indexing and image storing in the data base we search observed images.

\subsection{Segmentation}

In the method, to detect the blob, first of all, we use the color. Using blobs features we will classify them into different types of objects. The amount \& relation of those objects within the scene will let us for classify the images into different categories. To detect the blobs, we should first put the main image into a scale space and then by employing normalized laplacian $\nabla_{\text {norm }}^{2} l=\sigma^{2}\left(l_{x x}+l_{y y}\right)$ we show the blobs in a selected scale, at last, we label the regions obtained and classify the regions labeled. This stage, the blobs appear. So we should classify the color. To do this, we use MLP consists of six inputs, 11 outputs and bipolar sigmoidal functions. These inputs are the normalized red, green and blue values, the yellowness I, the purple $\mathrm{P}$ and the lightness $\mathrm{L}$ defined as follows:

$$
\begin{aligned}
\mathrm{L} & =\mathrm{R}+\mathrm{G}+\mathrm{B} \\
\mathrm{Y} & =(\mathrm{R}+\mathrm{G}) / 2 \\
\mathrm{P} & =(\mathrm{R}+\mathrm{B}) / 2 \\
\mathrm{r} & =\mathrm{R} / \mathrm{L} \\
\mathrm{g} & =\mathrm{G} / \mathrm{L} \\
\mathrm{b} & =\mathrm{B} / \mathrm{L}
\end{aligned}
$$

After classifying the colours, we extract their features. In this stage, after labelling each blob that we can volume geometric features and also extract any other factures of blob \& store them in the data bases of related image. Now its turn of obtaining correlation among detected blob edges. To do this we should use autocorregram (EOCA).

\subsection{Edge Orientation Auto Correlogram}

Correlogram is a proper tool to express the correlation between image elements. The EOAC classifies edges based on their orientation \& correlation between neighboring edges. This algorithm uses sober operator for edge detection. This operator generates two edges $\left(G_{x}, G_{y}\right)$ components. The amplitude \& edge orientation is computed as follows:

$$
\begin{gathered}
|G|=\sqrt{G_{x}^{2}+G_{y}^{2}} \\
G=\operatorname{tg}^{-1}\left(G_{y} \mid G_{x}\right)
\end{gathered}
$$

\subsection{Neural Network}

After extracting of existent blobs in image, using this features, grouping blobs to different categories that for this grouping we use MLP. After grouping blobs, images clustering to four categories: artificial, nature, face and text.

\subsection{Image Retrieval}

In the retrieval at first we extract the feature of desired image and then using this features we found relevant group to image and on that group search for image which have similar features with this image features. 


\section{Conclusions}

This paper by merging blob segmentations with feature vector of edge correlation \& color histogram could reduce any problems in indexing \& image retrieved in previous methods. Those improvements are as follow:

- There is no need to exact specify objects boundary.

- Instead of a pixel, blobs are our comparison tool.

- Recognition of images which blobs are combined is possible due to use of relationship feature vector.

- Between edges.

- Using of color histogram cause to retrieval be not turning.

- High speed of algorithm because of using classification.

- Acceptable results.

We used the matlab 6.5 environment for dismounting. The computer used in this research is Pentium IV and 2 $\mathrm{GHz}$ as extracting features from any pictures by employing this computer that lasts about 3 second, and also for establishing database, we assemble pictures from different sites in internet or take pictures by ourselves.

The applied pictures in this experiment are in 120*120 distances. For pictures with larger or smaller distances we must change the amount of distance to obtain on ideal amount. Algorithm of steps represented in Figures 1 and 2 for image indexing.

\section{Pluralization}

Image indexing and research is one of important context in the machine visual context. In the retrieval systems based on content, generally user's research is based on semantic features. Of course image indexing using low level features or general features is a difficult work. In this proposal method we have tried to use human visual system as a model, so we could extract any comprehendsible blobs from image which are comprehensible for human beings. So blob features are obtained for each vector image and stored in database. For improving the previous

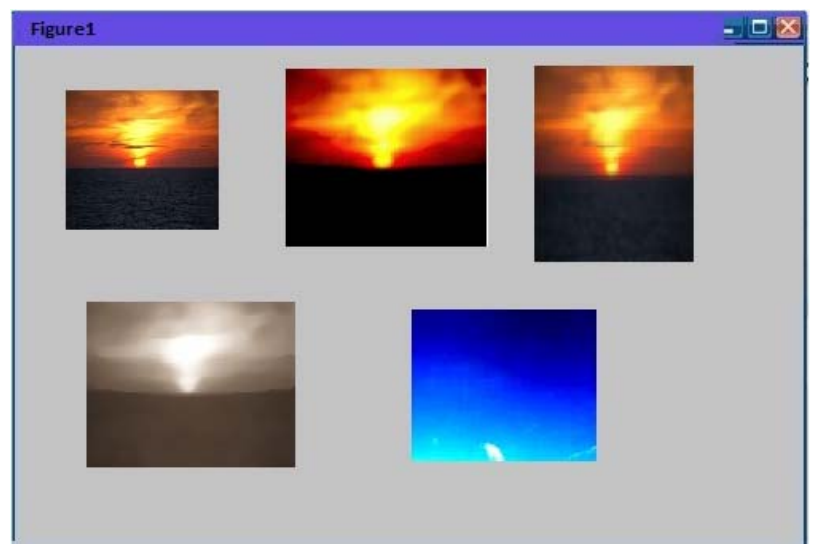

Figure 1. Indexing step for sunset picture.

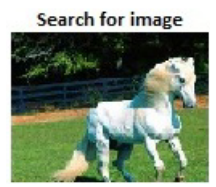

(a)

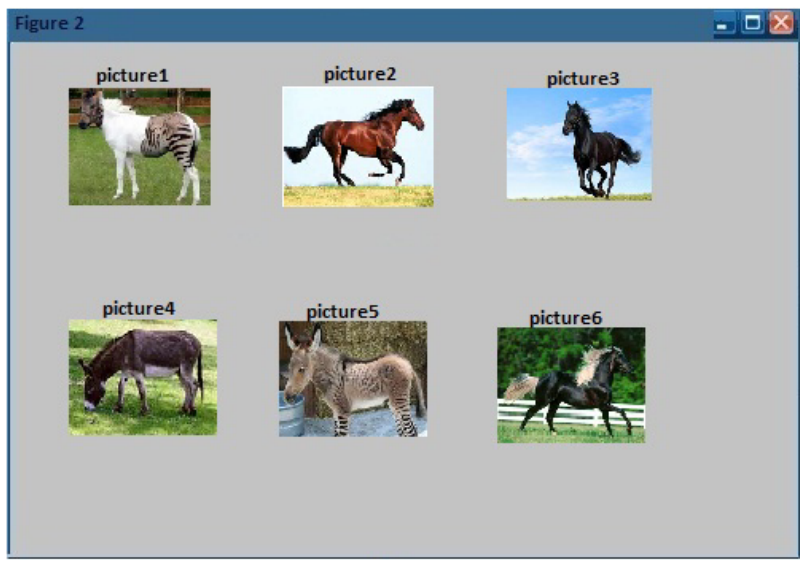

(b)

Figure 2. Getting results for horse picture a searched image b getting results.

methods, found edge \& color histogram are used too. In this proposed scheme we have employed about 1000 image on the database. Results of this research show that using blob which is inspiration of human vision and this blobs features and clustering we can raise speed and accuracy.

\section{REFERENCES}

[1] G. Iannizzotto, A. Puliafito and L. Vita, "Design and Implementation of a Content-Based Image Retrieval Tool," Proceedings of IEEE PDSE'97, Boston, 17-18 May 1997, pp. 304-310.

[2] H. Takahashi and M. Nakajima, "Graph-Based Color Image Segmentation Using Edge Magnitude for Image Understanding," Proceedings of International Workshop on Advanced Image Technology, February 2001, pp. 9396.

[3] W. Ma and B. Manjunath, "NeTra: A Toolbox for Navigating Large Images Databases,” ACM Multimedia Systems Journal, in Press.

[4] H. Takahashi and M. Nakajima, “Color Image Segmentation Using Region Growing Based on Neighbouring Region Features," Proceedings of International Workshop on Advanced Image Technology, Nagasaki, 21-22 January 2002, pp. 97-102.

[5] C. Carson, M. Thomas, S. Belongie, J. M. Hellerstein and J. Malik, "Blobworld: A System for Region-Based Image Indexing and Retrieval,” 3rd International Conference on Visual Information Systems, Amsterdam, 2-4 June 1999.

[6] J. Zheng and C. H. C. Leung, “Automatic Image Indexing for Rapid Content-Based Retrieval,” Proceedings of IEEE International Workshop on Multimedia Database Man- 
agement Systems, New York, 14-16 August 1996, pp. 38-45. doi:10.1109/MMDBMS.1996.541852

[7] A. K. Jain and A. Vailaya, "Shape-Based Retrieval: A Case Study with Trademark Image Database," Patter Recognition, Vol. 31, No. 9, 1998, pp. 1369-1390. doi:10.1016/S0031-3203(97)00131-3

[8] G. Qiu and S. Sudirman, “A Binary Color Vision Framework for Content Based Image Indexing,” School of Computer Science, The University of Nottingham, Nottingham, 2000.

[9] H. A. Moghaddam, T. T. Khajoie, A. H. Rouhi and M. S.
Tarzjan, "Wavelet Correlogram: A New Approach for Image Indexing and Retrieval,” 2005.

[10] M. Mirmehdi and R. Perissamy, "Perceptual Image Indexing and Retrieval," 2001.

[11] F. Mahmoudi, J. Shanbehzadeh, A.-M. Eftekhari-Moghadam and H. S. Zadeh, "A New Non-Segmentation ShapeBased Image Indexing Method,” 2003.

[12] D. G. Ruiz, H. Takahashi and M. Nakajima, "Image Categorization Using Color Blobs in a Mobile Environment," Eurographics, 2003. 